\title{
VALORACIÓN ECONÓMICA DE LA MORTALIDAD EN LA REGIÓN CARIBE DE COLOMBIA, 2004-2008*
}

\author{
Andrés Castaño Zuluaga** \\ Juan Correa Reyes ${ }^{* * *}$ \\ Luis Alvis Estrada**** \\ Nelson Alvis Guzmán ${ }^{* * * *}$
}

Recibido: febrero 15 de 2012 - Aceptado: mayo 24 de 2013

\section{RESUMEN}

El presente estudio estima el valor económico de la mortalidad en la región Caribe a través de la combinación del método de los años potenciales de vida perdidos, y el método del capital humano de valoración de la vida. Según los resultados obtenidos, los años potenciales de vida y la valoración económica de la mortalidad mostraron una tendencia decreciente de manera generalizada, explicada en gran parte por la disminución en las defunciones de los grupos de edad de menores de 1 año, y de 15 a 44 años que experimentó la región entre 2004 y 2008. El impacto económico de estas defunciones dentro de la producción departamental también mostró signos de decrecimiento, en su mayoría explicados por la tendencia alcista de la producción (PIB) en el ámbito departamental.

\section{PALABRAS CLAVE}

Economía de la salud, años potenciales de vida perdidos, valor de la vida.

\section{CLASIFICACIÓN JEL}

I12, J17.

\section{CONTENIDO}

Introducción, 1. Diseño metodológico; 2. Revisión de la literatura; 3. Descripción de la mortalidad en la región Caribe; 4. Años perdidos de vida potencial en el Caribe Colombiano; 5. Conclusiones; Bibliografía; Anexos.

\footnotetext{
Este artículo de investigación es producto de la tesis de grado de Andrés Mauricio Castaño Zuluaga, para optar por el titulo de economista en la Universidad de Cartagena. La investigación fue apoyada por el Grupo de Investigación de Economía de la Salud de la Universidad de Cartagena (GIES), clasificado en Al por Colciencias.

** Economista. Universidad de Cartagena, Colombia. Candidato a Magíster en Ciencia Regional, Universidad Católica del Norte, Chile. Correo electrónico: landres001@hotmail.com.

*** Economista. Candidato a Doctor en Educación. Miembro del grupo de investigación de Economía de la Salud de la Universidad de Cartagena, Colombia. Docente Facultad de Economía, Universidad de Cartagena, Colombia. Correo electrónico: profesorcorrea2011@hotmail.com.

**** Economista. Magíster Salud Pública. Miembro del grupo de investigación de Economía de la Salud de la Universidad de Cartagena. Docente Facultades de Enfermería y Medicina Universidad de Cartagena. Correo electrónico: lalvis20@yahoo.com.

${ }^{* * * * *}$ Médico. PhD. Economía de la Salud. Director del grupo de investigación de Economía de la Salud de la Universidad de Cartagena Docente Programa de Economía, Universidad de Cartagena, Colombia. Correo electrónico: nalvis20@yahoo.com.
} 


\section{ECONOMIC VALUATION OF MORTALITY IN THE CARIBBEAN REGION OF COLOMBIA \\ ABSTRACT}

This study estimates the economic value of mortality in the Caribbean region by the combination of two methods; potential years of life lost and human capital value of life. According to the obtained results, the potential years of life and the economic valuation for mortality have a generalized decreasing trend. This is mainly explained due to the reduction of deaths in infants less than one year old and ages between 15 and 44 years that the region has presented during the period 2004-2008. The economic impact of these deaths within departmental production also showed signs of decline, mostly explained by the upward trend in production (GDP) at the departmental level.

\section{KEY WORDS}

Health economics, years of potential life lost, the value of life

\section{JEL CLASSIFICATION}

I12, J17

\section{CONTENT}

Introduction, 1. Methodological design; 2. Literature review; 3. Mortality description of the Caribbean region; 4. Potential years of life lost in the Caribbean; 5. Conclusions; Bibliography; Attachments.

\section{VALORAÇÃO ECONOMICA DA MORTALIDADE NA REGIÃO CARIBE DA COLÔMBIA, 2004-2008 \\ RESUMO}

O presente estudo estima o valor económico da mortalidade na região Caribe por meio da combinação do método dos anos potências de vida perdidos e o método do capital humano de valoração da vida. Segundo os resultados obtidos; os anos potências de vida e a valoração económica da mortalidade mostraram uma tendência decrescente de maneira generalizada, explicada em grande parte pela diminuição nos óbitos dos grupos de idade de menores de 1 ano e de 15 a 44 anos que experimento a região entre 2004 e 2008. O impacto económico destes óbitos dentro da produção departamental também mostrou signos de decrescimento, em sua maioria explicada pela tendência de aumento da produção (PIB) no âmbito departamental.

\section{PALAVRAS CHAVE}

Economia da saúde, anos potenciais de vida perdidos, valor da vida.

\section{CLASIFICAÇÃO JEL}

I12, J17.

\section{CONTEÚDO}

Introdução, 1. Desenho metodológico; 2. Revisão de literatura; 3. Descrição da mortalidade na região Caribe; 4. Anos perdidos de vida potencial no Caribe Colombiano; 5. Conclusões; Bibliografia; Anexos. 
Valoración económica de la mortalidad en la región Caribe de Colombia, 2004-2008

\section{INTRODUCCIÓN}

En Colombia, así como en cualquier país del mundo, el nivel de mortalidad es un indicador asociado con la calidad de vida, la seguridad, el nivel de bienestar y el estado de salud de la población. Altas tasas de mortalidad, sobre todo en la población joven en alguna región o país, también son un buen parámetro para medir la capacidad de relevo que tiene el mercado laboral. Más importante aún, es que altos niveles de mortalidad en la población joven representan a largo plazo una pérdida de capital humano, que afecta el consumo y condiciona el crecimiento esperado de la economía.

Una de las herramientas para medir dicha pérdida de capital humano es la valoración económica de la mortalidad, la cual procura expresar en términos de ingresos de por vida lo que dejan de recibir las personas a causa de una muerte prematura (mucho antes de su expectativa de vida). Para determinar el monto de los ingresos que deja de recibir la persona es necesario calcular los años potenciales de vida perdidos (APVP) ${ }^{1}$. De igual modo, para los programas públicos, dedicados el control, la erradicación de la enfermedad y la disminución de mortalidad por ciertas causas específicas, la valoración de la vida humana (mortalidad) es un requisito indispensable para el cálculo correcto de los beneficios o resultados derivados de alguna intervención de política pública. Lo anterior adquiere importancia cuando se entiende que la mortalidad es un fenómeno con una distribución heterogénea a lo largo de las regiones y departamentos colombianos, por lo cual, la valoración económica de esas muertes en los distintos niveles puede contribuir a implementar políticas públicas mejor dirigidas y más efectivas en la erradicación de la enfermedad y la disminución de la mortalidad.

Para el caso de Colombia, la mortalidad es un fenómeno con una distribución geográfica desigual a lo largo de las regiones. La región Caribe es un ejemplo de esta situación. Según cálculos propios basados en los resultados del Censo General de 2005 suministrados por el DANE, la región Caribe², representó cerca del 16 \% de las defunciones no fetales totales en el país, cuando en términos de población agrupa a un poco más del 21 \%, la región Andina, por su parte, representa el 56 \% de la población, pero sus defunciones alcanzan el 60 \% del total; asimismo, la Pacífica que agrupa el 20\% de las defunciones del país, solo representa el 17\% de la población del total. Esta situación, sumada a las características geográficas,

\footnotetext{
APVP son años potenciales de vida perdidos, estos son un ingrediente para el cálculo de la valoración económica de la mortalidad, dado que indican la cantidad de años que una persona perdió por motivo de una defunción acontecida en un momento anterior a lo que dictaminaba su expectativa de vida.

2 La conformación de la región Caribe se hizo de acuerdo la división política administrativa de Colombia. Según esta, la región Caribe está conformada por 8 departamentos: Atlántico, Bolívar, Cesar, Córdoba, La Guajira, Magdalena, Sucre y San Andrés y Providencia.
} 
culturales y socio-económicas de cada región, exige que la estimación del valor económico de las muertes sea evaluada de manera más desagregada, teniendo en cuenta departamento, sexo y causas agrupadas de defunción.

El objetivo de este documento es ofrecer estimaciones, por sexo, de los años potenciales de vida perdidos para los departamentos de la región Caribe, y del valor presente de los ingresos perdidos de toda la vida para los departamentos de la región caribe. Esta información suministrará a los decisores de política pública, un diagnóstico del impacto económico real de las defunciones en ciertos grupos de edad en los departamentos de la región Caribe, lo que hará más sencilla la intervención o la formulación de políticas públicas encaminadas a menguar las causas de defunción más nocivas para la conservación de la vida.

Además de esta introducción, el documento está organizado en cinco secciones. En la primera sección, se presenta una revisión de la literatura respecto al método del capital humano de valoración de la vida y de los estudios de mortalidad que han empleado los años potenciales de vida perdidos tanto en la esfera nacional, como en la internacional. La segunda sección describe los datos utilizados y la metodología empleada. En la tercera sección, se presenta una descripción de la mortalidad en el ámbito nacional y departamental teniendo en cuenta el sexo, grupos de edad y causas agrupadas de defunción. En la sección cuatro, se exhiben los resultados de las estimaciones de los APVP y su valoración económica. Finalmente, en la sección cinco se presentan las conclusiones del estudio.

\section{REVISIÓN DE LA LITERATURA}

La mortalidad es, dentro de los indicadores socio-demográficos, el que mejor expresa los niveles de vida y pobreza, en particular la mortalidad infantil. Su interrelación con el bienestar económico es sostenida desde hace mucho tiempo. Landry (1934) afirma en su teoría de la transición demográfica, que sin mejoras del bienestar no hubiese sido posible disminuir la mortalidad infantil. Es así como Cruz y Muñoz ( 2005) señalan que desde el punto de vista económico, se han hecho intentos por obtener una valoración de la vida y de la muerte a través del método del capital humano de valoración de la vida, el cual establece que el valor de la vida humana se calcula con base en lo que la persona en cuestión deja de producir si fallece, teniendo en cuenta, entre otros aspectos, los salarios y el consumo personal corregido por la tasa social de descuento, es decir, lo que va a producir en el resto de sus días, menos lo que consuma.

Si bien el método del capital humano de valoración de la vida ha sido utilizado con mucha frecuencia en tiempos recientes, la cuantificación del valor de la vida 
Valoración económica de la mortalidad en la región Caribe de Colombia, 2004-2008

humana en términos económicos no es un concepto nuevo. Desde el siglo XVII con los aportes realizados por Petty (1690), siglos más tarde con los de Smith (1776), y Farr (1853), se comenzaron a gestar metodologías para la valoración económica de la vida humana, y autores como Dublín y Lotka (1934) utilizaron las ideas aportadas por Petty (1960) y Farr (1853), en el diseño de un procedimiento para valorar en el sentido económico el trabajo de los esclavos en la Edad Media, hasta llegar a estudios como el de Dempsey (1947), Dickinson y Welker (1948) y Haenszel (1950).

Sin embargo, la valoración económica de la vida para medir los ingresos potenciales perdidos por una persona que fallece antes de lo que dictamina su expectativa de vida, solo se comienza a emplear a partir del desarrollo de estudios del impacto de la mortalidad. Arriaga (1984), teniendo en cuenta la creación de una metodología para el cálculo de los APVP, muestra que estos se pueden utilizar principalmente para la elaboración de la descomposición del cambio en la esperanza de vida, dado que permiten medir la contribución de cada causa de muerte y/o grupo de edad al cambio observado en la esperanza de vida. Es así como los APVP no solo permiten valorar los cambios en la mortalidad sino también la relevancia de algún tipo de defunciones dentro de la mortalidad global.

Los APVP se calculan a través de las pérdidas que sufre la sociedad como consecuencia de la muerte de personas jóvenes o de fallecimientos prematuros. La Organización Panamericana de la Salud (OPS), define una muerte prematura como aquella que ocurre antes de lo que dictamina la esperanza de vida de la población bajo estudio. La ventaja de usar la edad a la cual mueren las personas y no el evento de muerte en sí mismo es que se puede ponderar de modo distinto los eventos de mortalidad que ocurren en diferentes momentos de la vida. Los APVP asumen que en la medida en que la muerte sea a una edad menor, mayor es la pérdida de ese evento. De acuerdo con la OPS, los APVP de una determinada causa de muerte son la sumatoria a través de todas las personas que fallecen por una causa en específico, de los años que debieran haber vivido, si fallecieran de acuerdo con su expectativa de vida particular. El objetivo del indicador es mostrar de modo amplio la importancia relativa de las causas de defunción más trascendentes de mortalidad prematura, y su principal uso se da en la esfera de la planificación y definición de prioridades en salud.

El análisis de los años potenciales de vida perdidos suele ser dirigido a causas específicas o grupo de causas en alguna unidad territorial, sin aplicar el método del capital humano para la valoración económica sobre estos valores obtenidos. Lo anterior implica que el uso de los APVP solo da idea de la contribución de cada enfermedad a los cambios experimentados en la expectativa de vida, pero no puede decir nada respecto al impacto económico de una enfermad en específico 
sobre una población. En este contexto se destacan en la esfera internacional las investigaciones de Arriaga (1996), Bocco (1996), Bertone y otros (2009), Boleda y Arriaga (2000), Bustamante (1994) y Pérez y otros (2009). En la esfera nacional, las investigaciones que involucran el cálculo de años potenciales de vida perdidos también son diversas. Algunas los utilizan para medir los cambios en la mortalidad por sexo y grupo de causas y su efecto sobre la esperanza de vida (López y Arce, 2008); otros se limitan a calcularlos de acuerdo con la mortalidad de una unidad territorial en particular (Toro y otros, 2007).

En Colombia, pocos autores han intentado relacionar el comportamiento de los años potenciales de vida perdidos con la valoración económica de la mortalidad a través del método del capital humano, entre los cuales se destacan Alvis y Alvis (2009), Alvis y De La Hoz (2004) y Alvis y otros (2009). La principal deficiencia en la bibliografía colombiana en lo que respecta a este ítem es que la valoración económica tiende hacerse, ya sea solo para una enfermedad o para un grupo de enfermedades, ya sea para un municipio, mas no para una región en particular como lo aborda el presente estudio.

\section{DISEÑO METODOLÓGICO}

Para estudiar el fenómeno de la mortalidad en la región Caribe, se utilizan los registros de estadísticas vitales para cada departamento, publicados por el Departamento Administrativo Nacional de Estadísticas (DANE). De allí, se extraen: el número de defunciones no fetales por sexo, grupo etario y causas agrupadas (según la lista 6/67-CIE-10 OMS/OPS) para los departamentos de Atlántico, Bolívar, Cesar, Córdoba, La Guajira, Magdalena, Sucre y San Andrés y Providencia.

Cabe destacar que la clasificación internacional de enfermedades atribuibles a las defunciones no fetales (CIE-10) agrupa las diferentes contrariedades de salud en siete grupos exhaustivos y excluyentes: Grupo 1. Enfermedades transmisibles; Grupo 2. Neoplastias (tumores); Grupo 3. Enfermedades del sistema circulatorio; Grupo 4: Afecciones originadas en el periodo perinatal; Grupo 5.Causas externas; Grupo 6: Todas las demás causas; Grupo 7. Síntomas, signos y afecciones mal definidas.

Para el cálculo de los años potenciales de vida perdidos se sigue el procedimiento propuesto por Arriaga (1996). Los APVP para una causa definida se definen como la sumatoria de las defunciones por esa causa en cada grupo de edad, y luego, se multiplica el resultado por los años que restan desde la edad central del grupo etario hasta la edad límite considerada, en términos formales:

$$
\mathrm{APVP}=\sum_{i=1}^{\mathrm{L}}\left[(\mathrm{L}-i) * d_{i}\right]
$$


Valoración económica de la mortalidad en la región Caribe de Colombia, 2004-2008

Donde I es la edad límite inferior establecida, L es la edad límite superior establecida, i es la edad de la muerte y $d_{i}$ es el número de defunciones de la edad i. Para obtener el valor de los APVP expresado en índice (IAPVP) se utiliza la siguiente ecuación:

$$
\operatorname{IAPVP}_{i}=\left(\operatorname{APVP}_{i} / \mathrm{N}_{i}\right) * 1000
$$

Donde $\mathrm{N}$ es la población comprendida entre el límite inferior y el superior de las edades estudiadas y el subíndice i indica que los cálculos se pueden hacer para los valores obtenidos de años potenciales de vida perdidos de cada grupo poblacional.

En lo concerniente a la utilización de los APVP para evaluar la importancia de la mortalidad, se asume la hipótesis de que una defunción prematura provoca una pérdida mayor cuanto más joven es la persona que fallece. Por tanto, los APVP más inmediatos tienen un mayor valor, y estos disminuyen de acuerdo con una tasa de descuento (que permita involucrar las muertes de adultos y ancianos). De acuerdo al método de capital humano de valoración de la vida humana, el valor presente del número de años perdidos a los que se les aplica el descuento "r", se plantea así:

$$
\operatorname{APVAP}_{i}=\operatorname{APVP}_{i}(1+r)^{n-1}
$$

Donde APVAP ${ }_{i}$ son años potenciales de vida perdidos ajustados al año i, APVP ${ }_{i}$ es el número de años perdidos a los que se aplica el descuento para cada año del período de estudio, r es la tasa de descuento o actualización expresada en porcentaje. En este caso, $r=3 \%{ }^{3}$, n son los años del período de estudio.

El procedimiento para el cálculo de la valoración económica de las pérdidas fue el siguiente: se tomó el salario mensual de los registros oficiales del Departamento Administrativo Nacional de Estadísticas (DANE) sobre el valor del Salario Mínimo Legal Vigente (SMLV) y para cada año de estudio se multiplicó por los 12 meses para determinar el monto de ingresos o consumos mínimos anuales. Luego, se tomó la base de precios del DANE ajustada a diciembre de 2005 y se adaptó al año base del estudio (2008). Posteriormente, se llevaron a pesos constantes del año final del período los salarios mínimos anuales de los años estudiados, para determinar el valor anual en pesos constantes de diciembre del año base del estudio, que para este caso es 2008, y se estableció el total de años perdidos de cada período anual (obtenidos a través del método del capital humano de valoración de la vida), para

3 Uno de los temas más importantes a la hora de utilizar el método de capital humano de valoración de la vida humana, es la definición de la tasa de descuento a utilizar. En este estudio se utilizara la tasa promedio encontrada en los trabajos metodológicamente parecidos, que oscila entre el 3\% y 5\%. 
luego multiplicarlos por el ingreso mínimo obtenido, y en la etapa final, se efectuó la sumatoria de los flujos anuales a pesos constantes del año base con el fin de determinar el valor total del costo de los años de vida perdidos en el período.

\section{LA MORTALIDAD EN LA REGIÓN CARIBE DE COLOMBIA, 2004-2008}

El nivel de defunciones totales de la región Caribe presentó una tendencia creciente pero moderada. El promedio de defunciones para la región Caribe durante el periodo 2004-2008, fue de 31.252. La variabilidad de las defunciones, expresada a través de la desviación que experimenta el nivel de mortalidad de la media obtenida para el periodo, es moderada. Solamente en los años 2004 y 2008 se presentan las mayores variaciones con respecto a la media de las defunciones con $-2,38$ \% y 2,83 $\%$ respectivamente (tabla 1 ).

Tabla 1. Mortalidad, población y tasa bruta de mortalidad para los departamentos de la región Caribe, 2004-2008.

\begin{tabular}{|c|c|c|c|c|c|c|c|c|c|}
\hline \multirow[b]{2}{*}{ Depart. } & \multirow[b]{2}{*}{ Año } & \multicolumn{3}{|c|}{ Mortalidad } & \multicolumn{3}{|c|}{ Población } & \multicolumn{2}{|c|}{ TBM } \\
\hline & & Muertes & $\begin{array}{c}\text { Partici- } \\
\text { pación } \\
\text { Regional } \\
\text { (\%) }\end{array}$ & $\begin{array}{c}\text { Tasa (\%) } \\
\text { crecimien- } \\
\text { to }\end{array}$ & Población & $\begin{array}{l}\text { Partici- } \\
\text { pación } \\
\text { Regional } \\
(\%)\end{array}$ & $\begin{array}{c}\text { Tasa (\%) } \\
\text { crecimiento }\end{array}$ & TBM & $\begin{array}{c}\text { Tasa (\%) } \\
\text { crecimiento }\end{array}$ \\
\hline \multirow{5}{*}{ Atlántico } & 2004 & 8.940 & 29,3 & & 2.136 .041 & 23,8 & & 4,2 & \\
\hline & 2005 & 8.845 & 28,7 & $-1,1$ & 2.166 .156 & 23,8 & 1,41 & 4,1 & $-2,4$ \\
\hline & 2006 & 9.210 & 29,4 & 4,1 & 2.195 .778 & 23,8 & 1,37 & 4,2 & 2,7 \\
\hline & 2007 & 8.805 & 28,0 & $-4,4$ & 2.225 .462 & 23,8 & 1,35 & 4,0 & $-5,7$ \\
\hline & 2008 & 8.958 & 27,9 & 1,7 & 2.255 .164 & 23,8 & 1,33 & 4,0 & 0,4 \\
\hline \multirow{5}{*}{ Bolívar } & 2004 & 5.565 & 18,2 & & 1.861 .487 & 20,8 & & 3,0 & \\
\hline & 2005 & 5.919 & 19,2 & 6,4 & 1.878 .993 & 20,7 & 0,94 & 3,2 & 5,4 \\
\hline & 2006 & 5.952 & 19,0 & 0,6 & 1.897 .658 & 20,6 & 0,99 & 3,1 & $-0,4$ \\
\hline & 2007 & 5.251 & 16,7 & $-11,8$ & 1.917 .112 & 20,5 & 1,03 & 2,7 & $-12,7$ \\
\hline & 2008 & 6.086 & 18,9 & 15,9 & 1.937 .316 & 20,4 & 1,05 & 3,1 & 14,7 \\
\hline \multirow{5}{*}{ Cesar } & 2004 & 3.257 & 10,7 & & 891.089 & 9,9 & & 3,7 & \\
\hline & 2005 & 3.204 & 10,4 & $-1,6$ & 903.279 & 9,9 & 1,37 & 3,5 & $-3,0$ \\
\hline & 2006 & 3.108 & 9,9 & $-3,0$ & 915.923 & 9,9 & 1,40 & 3,4 & $-4,3$ \\
\hline & 2007 & 3.367 & 10,7 & 8,3 & 928.569 & 9,9 & 1,38 & 3,6 & 6,9 \\
\hline & 2008 & 3.674 & 11,4 & 9,1 & 941.207 & 9,9 & 1,36 & 3,9 & 7,7 \\
\hline
\end{tabular}


Valoración económica de la mortalidad en la región Caribe de Colombia, 2004-2008

\begin{tabular}{|c|c|c|c|c|c|c|c|c|c|}
\hline \multirow[b]{2}{*}{ Depart. } & \multirow[b]{2}{*}{ Año } & \multicolumn{3}{|c|}{ Mortalidad } & \multicolumn{3}{|c|}{ Población } & \multicolumn{2}{|c|}{ TBM } \\
\hline & & Muertes & $\begin{array}{l}\text { Partici- } \\
\text { pación } \\
\text { Regional } \\
(\%)\end{array}$ & $\begin{array}{c}\text { Tasa (\%) } \\
\text { crecimien- } \\
\text { to }\end{array}$ & Población & $\begin{array}{l}\text { Partici- } \\
\text { pación } \\
\text { Regional } \\
\text { (\%) }\end{array}$ & $\begin{array}{c}\text { Tasa (\%) } \\
\text { crecimiento }\end{array}$ & TBM & $\begin{array}{c}\text { Tasa (\%) } \\
\text { crecimiento }\end{array}$ \\
\hline \multirow{5}{*}{ Córdoba } & 2004 & 4.489 & 14,7 & & 1.446 .416 & 16,1 & & 3,1 & \\
\hline & 2005 & 4.460 & 14,5 & $-0,6$ & 1.467 .929 & 16,1 & 1,49 & 3,0 & $-2,1$ \\
\hline & 2006 & 4.467 & 14,3 & 0,2 & 1.489 .669 & 16,2 & 1,48 & 3,0 & $-1,3$ \\
\hline & 2007 & 4.835 & 15,3 & 8,2 & 1.511 .981 & 16,2 & 1,50 & 3,2 & 6,6 \\
\hline & 2008 & 4.678 & 14,6 & $-3,2$ & 1.535 .375 & 16,2 & 1,55 & 3,0 & $-4,7$ \\
\hline \multirow{5}{*}{ La Guajira } & 2004 & 1.579 & 5,2 & & 653.702 & 7,3 & & 2,4 & \\
\hline & 2005 & 1.692 & 5,5 & 7,2 & 681.575 & 7,5 & 4,26 & 2,5 & 2,8 \\
\hline & 2006 & 1.588 & 5,1 & $-6,1$ & 708.673 & 7,7 & 3,98 & 2,2 & $-9,7$ \\
\hline & 2007 & 1.692 & 5,4 & 6,5 & 735.974 & 7,9 & 3,85 & 2,3 & 2,6 \\
\hline & 2008 & 1.648 & 5,1 & $-2,6$ & 763.439 & 8,1 & 3,73 & 2,2 & $-6,1$ \\
\hline \multirow{5}{*}{$\begin{array}{l}\text { Magda- } \\
\text { lena }\end{array}$} & 2004 & 3.973 & 13,0 & & 1.140 .241 & 12,7 & & 3,5 & \\
\hline & 2005 & 4.046 & 13,1 & 1,8 & 1.149 .917 & 12,6 & 0,85 & 3,5 & 1,0 \\
\hline & 2006 & 4.193 & 13,4 & 3,6 & 1.159 .729 & 12,6 & 0,85 & 3,6 & 2,8 \\
\hline & 2007 & 4.526 & 14,4 & 7,9 & 1.169 .770 & 12,5 & 0,87 & 3,9 & 7,0 \\
\hline & 2008 & 4.307 & 13,4 & $-4,8$ & 1.180 .051 & 12,4 & 0,88 & 3,6 & $-5,7$ \\
\hline \multirow{5}{*}{$\begin{array}{l}\text { San } \\
\text { Andrés }\end{array}$} & 2004 & 210 & 0,7 & & 69.920 & 0,8 & & 3,0 & \\
\hline & 2005 & 224 & 0,7 & 6,7 & 70.554 & 0,8 & 0,91 & 3,2 & 5,7 \\
\hline & 2006 & 215 & 0,7 & $-4,0$ & 71.075 & 0,8 & 0,74 & 3,0 & $-4,7$ \\
\hline & 2007 & 227 & 0,7 & 5,6 & 71.613 & 0,8 & 0,76 & 3,2 & 4,8 \\
\hline & 2008 & 210 & 0,7 & $-7,5$ & 72.167 & 0,8 & 0,77 & 2,9 & $-8,2$ \\
\hline \multirow{5}{*}{ Sucre } & 2004 & 2.493 & 8,2 & & 764.453 & 8,5 & & 3,3 & \\
\hline & 2005 & 2.435 & 7,9 & $-2,3$ & 772.010 & 8,5 & 0,99 & 3,2 & $-3,3$ \\
\hline & 2006 & 2.555 & 8,2 & 4,9 & 779.532 & 8,5 & 0,97 & 3,3 & 3,9 \\
\hline & 2007 & 2.798 & 8,9 & 9,5 & 787.167 & 8,4 & 0,98 & 3,6 & 8,4 \\
\hline & 2008 & 2.577 & 8,0 & $-7,9$ & 794.904 & 8,4 & 0,98 & 3,2 & $-8,8$ \\
\hline \multirow{5}{*}{ Región } & 2004 & 30.506 & 100,0 & & 8.963 .349 & 100,0 & & 3,4 & \\
\hline & 2005 & 30.825 & 100,0 & 1,0 & 9.090 .413 & 100,0 & 1,42 & 3,4 & $-0,4$ \\
\hline & 2006 & 31.288 & 100,0 & 1,5 & 9.218 .037 & 100,0 & 1,40 & 3,4 & 0,1 \\
\hline & 2007 & 31.501 & 100,0 & 0,7 & 9.347 .648 & 100,0 & 1,41 & 3,4 & $-0,7$ \\
\hline & 2008 & 32.138 & 100,0 & 2,0 & 9.479 .623 & 100,0 & 1,41 & 3,4 & 0,6 \\
\hline
\end{tabular}

Fuente: elaboración de los autores con base en DANE - Estadísticas vitales 
En el ámbito departamental, en la región Caribe, Atlántico, Bolívar, Córdoba y Magdalena aportan la mayor cantidad de defunciones. Otros, como San Andrés y La Guajira son los que registran la menor cantidad de defunciones. Atlántico, a pesar de ser el departamento con mayor participación dentro de las defunciones, mantuvo una senda de crecimiento casi invariable de 2004 a 2008; este fenómeno provocó un cambio en la estructura de mortalidad de la región, donde departamentos como Bolívar, Cesar y Magdalena cada vez ganan más participación.

Gráfico 1. Tasa especifica de mortalidad por sexo región Caribe, 2004-2008

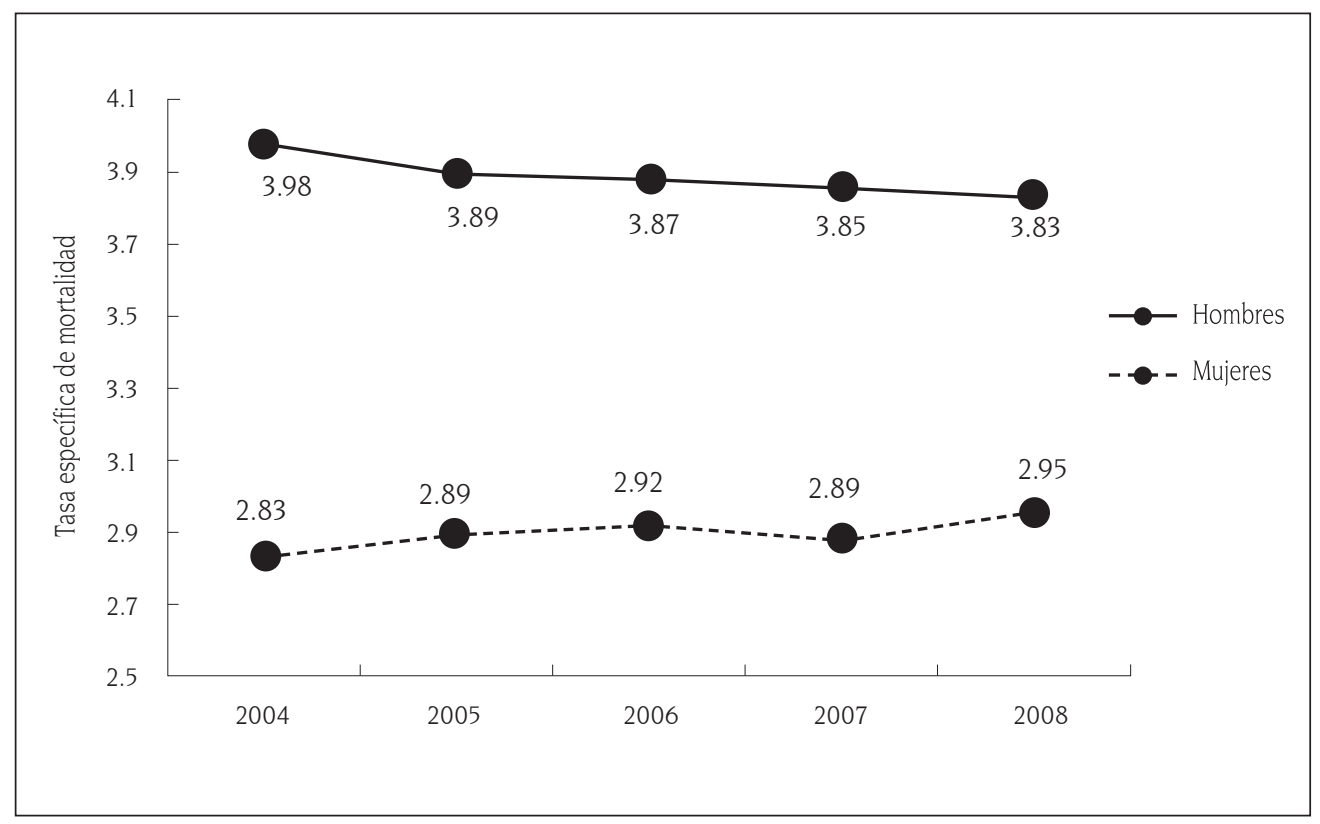

Fuente: elaboración de los autores con base en DANE - Estadísticas Vitales.

Por sexo (gráfico 1), el comportamiento de la tasa específica de mortalidad (TEM) en la región Caribe para el período 2004-2008 ha tendido a aumentar en las mujeres y a disminuir en los hombres. La tasa específica de mortalidad por sexo en las mujeres, creció cerca de 0,12 muertes por cada mil habitantes, mientras que en los hombres la TEM disminuyó en 0,15 defunciones por cada mil habitantes en el período 2004-2008. A pesar de que las defunciones, tanto de hombres como de mujeres, crecieron para el período de estudio, la intensidad con que lo hicieron fue distinta. Las defunciones en hombres crecieron a una tasa promedio para el período de $0,33 \%$, mientras que las defunciones en mujeres crecieron a una tasa promedio de 2,23\%. 
Gráfica 2. Defunciones de hombres por departamento de la región Caribe, 2004-2008

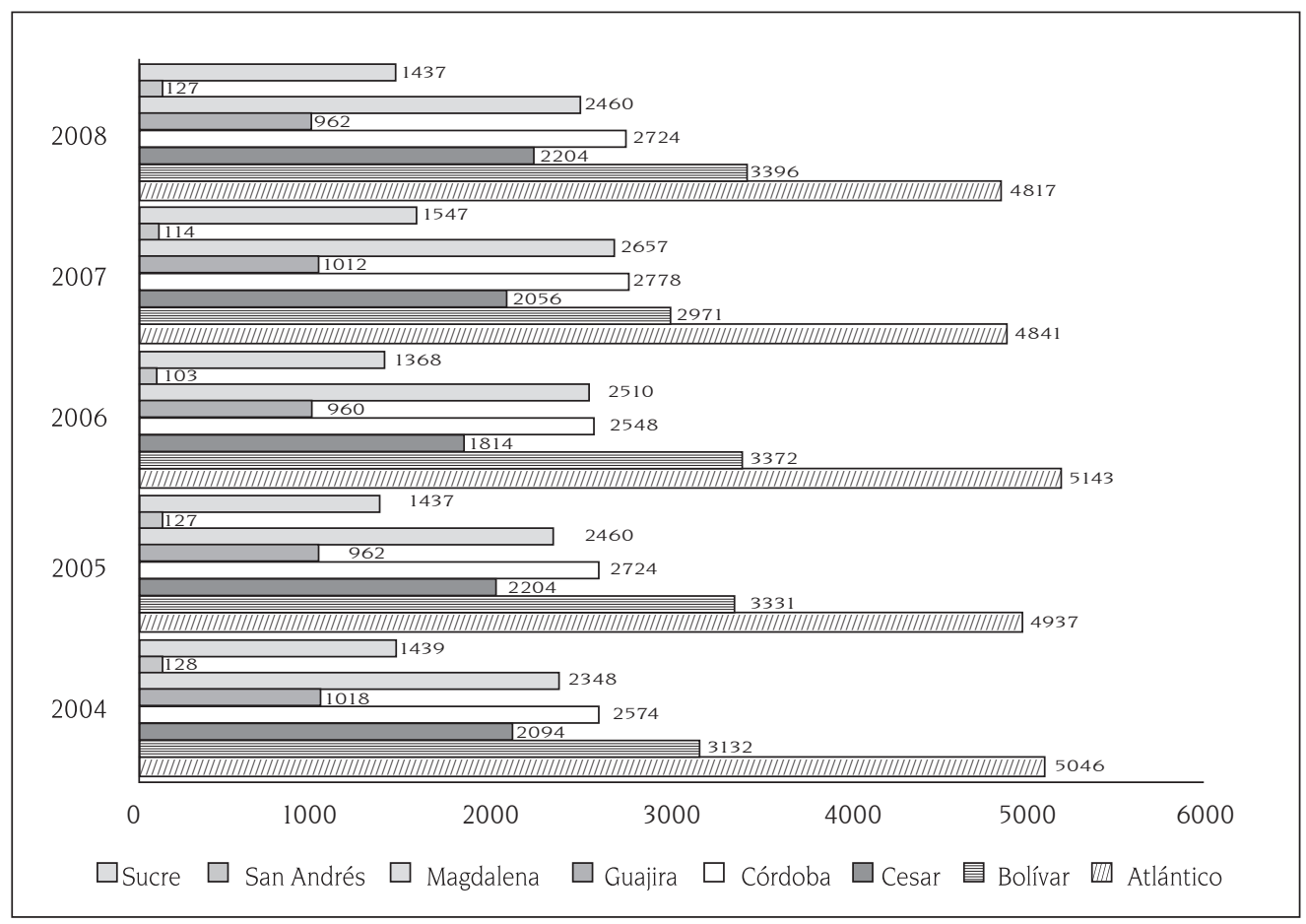

Fuente: elaboración de los autores con base en DANE - Estadísticas vitales

Las defunciones en hombres por departamento (gráfico 2) muestran una tendencia a decrecer en departamentos como Atlántico y La Guajira, una tendencia a aumentar en departamentos como Bolívar, Córdoba, Cesar y Magdalena, y una tendencia estable para el caso de San Andrés y Sucre. En Atlántico, Las defunciones de hombres disminuyeron alrededor de 4,6 \% del año 2004 al año 2008, pasando de 5.046 defunciones en 2004 hasta 4.817 en 2008. Por otro lado, en lo que concierne a los departamentos con una trayectoria de crecimiento positiva, se destacan la conducta de Bolívar, Cesar y Córdoba, para las cuales los eventos de mortalidad masculina crecieron entre 2004 y 2008 en 8,42 \%, 5,25 \% y 5,82 \%, respectivamente.

Por el lado de las mujeres, la mortalidad parece seguir un patrón distinto al de los hombres (gráfico 3). Durante el período, los eventos de mortalidad presentaron aumentos bastante significativos en departamentos como Bolívar, Magdalena, Cesar y Guajira. Para el caso de Bolívar y Magdalena, el crecimiento de las defunciones estuvo alrededor del 10 \%. No obstante, Cesar y La Guajira fueron, de lejos, los departamentos que generaron mayor intranquilidad, dado que la mortalidad en mujeres creció exageradamente en 26,4 \% y 22,3 \%, respectivamente, entre 2004 
y 2008. Otros departamentos como Atlántico y Sucre, presentaron crecimientos más moderados (6,35 \% y 8,16 \%). Finalmente, San Andrés y Córdoba, a pesar de mantener tasas de crecimiento positivas para el periodo, estas fueron mucho menos dinámicas que las del resto de departamentos (1,22 \% y 2,09\%, respectivamente).

Gráfico 3: Defunciones de mujeres por departamento de la región Caribe, 2004-2008

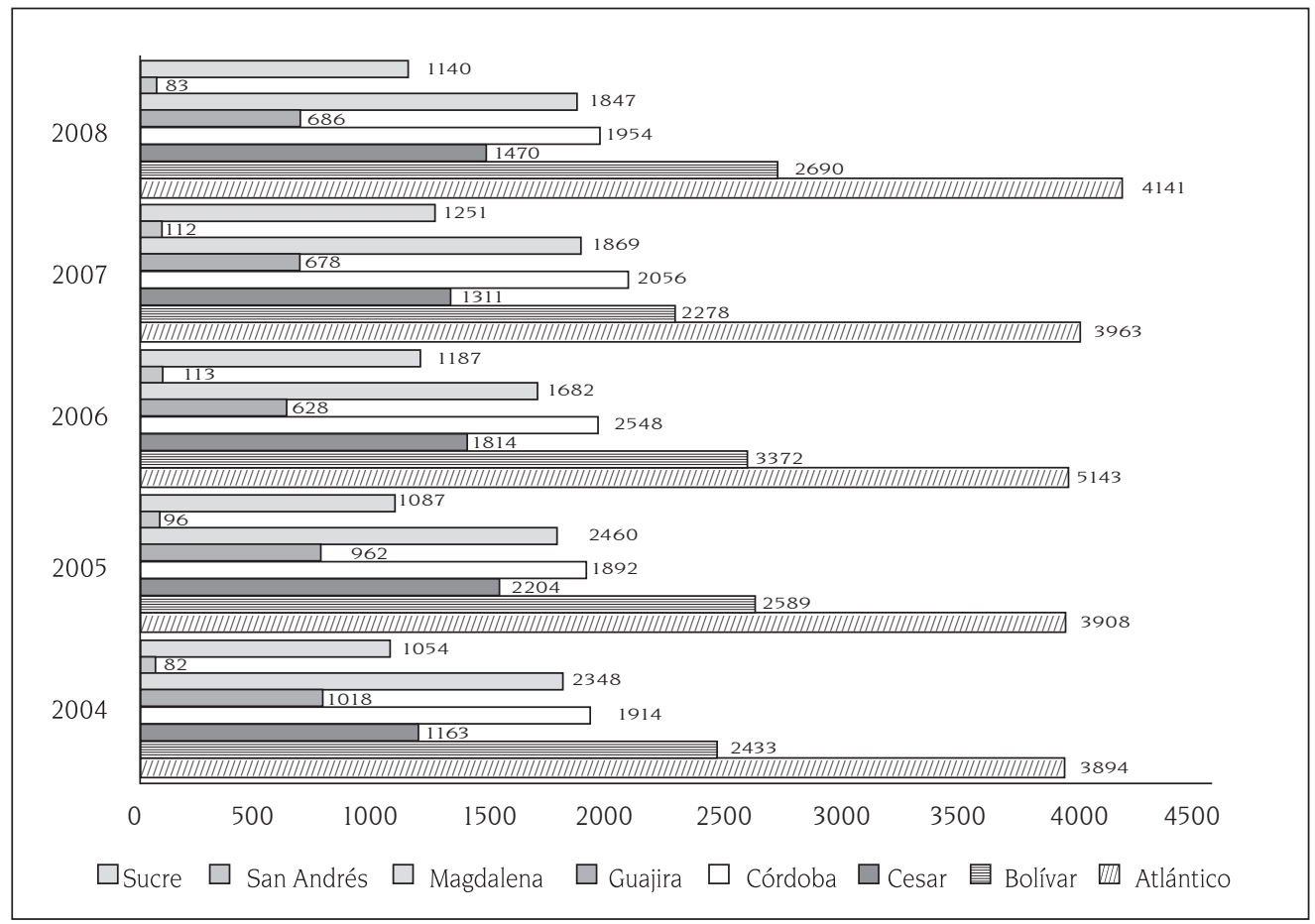

Fuente: Elaboración de los autores con base en DANE - Estadísticas Vitales.

Una mejor forma de entender el comportamiento de los eventos de mortalidad en la esfera departamental es si se analiza la mortalidad de acuerdo con causas agrupadas (ver anexo 2). Departamentos como Atlántico, Bolívar, Cesar y Magdalena exhiben un preocupante aumento de las defunciones por neoplasias entre el año 2004 y el 2008. Córdoba debe poner especial énfasis en la mortalidad relacionada con eventos externos, mientras que Sucre muestra cifras preocupantes de eventos relacionados con mortalidad infantil. Las muertes por afecciones perinatales crecieron $58 \%$ entre 2004 y 2008.

Atlántico, Cesar, La Guajira, Magdalena, San Andrés y Sucre lograron disminuir los eventos de mortalidad relacionados con causas externas de una manera sostenida entre 2004 y 2008. En términos absolutos, la mayor disminución la experimentó 
Valoración económica de la mortalidad en la región Caribe de Colombia, 2004-2008

Atlántico con 376 defunciones menos por esta causa. En términos relativos, La Guajira, Atlántico y Sucre lograron reducciones del orden del 30 \%, en Cesar y Magdalena fue del orden de $20 \%$ y $11 \%$, respectivamente.

\section{AÑOS POTENCIALES DE VIDA PERDIDOS EN EL CARIBE COLOMBIANO, 2004-2008}

Uno de los objetivos de este trabajo es calcular los años potenciales de vida perdidos (APVP), dado que sirven para monitorear el impacto de cierto grupo de enfermedades en determinada población, y tienen una ventaja con respecto a los índices brutos; reflejan la mortalidad en los grupos de edad más temprano, asignando mayor peso a las muertes que ocurren en los más jóvenes. Esto, en términos económicos, tiene importancia, dado que en la medida en que se identifiquen causas de muerte en edades tempranas se pueden formular políticas de salud más efectivas para menguar la carga de dichas afecciones y, asimismo, darle al aparato económico y al mercado laboral posibilidades de renovación.

Tabla 2. APVP por sexo en los departamentos de la región Caribe, 2004-2008

\begin{tabular}{|c|c|c|c|c|c|c|c|c|c|c|}
\hline \multicolumn{11}{|c|}{ Hombres } \\
\hline \multirow{2}{*}{ Departamento } & \multicolumn{2}{|c|}{2004} & \multicolumn{2}{|c|}{2005} & \multicolumn{2}{|c|}{2006} & \multicolumn{2}{|c|}{2007} & \multicolumn{2}{|c|}{2008} \\
\hline & APVP & Part. \% & APVP & Part. \% & APVP & Part. \% & APVP & Part. \% & APVP & Part. \% \\
\hline Atlántico & 96.919 & 25,2 & 90.801 & 25,1 & 88.480 & 25,9 & 78.624 & 23,9 & 70.836 & 22,6 \\
\hline Bolívar & 66.629 & 17,3 & 69.158 & 19,1 & 62.283 & 18,2 & 52.461 & 15,9 & 57.473 & 18,3 \\
\hline Cesar & 52.083 & 13,5 & 46.558 & 12,9 & 40.581 & 11,9 & 45.331 & 13,8 & 44.779 & 14,3 \\
\hline Córdoba & 55.786 & 14,5 & 53.680 & 14,8 & 47.197 & 13,8 & 49.277 & 15,0 & 52.314 & 16,7 \\
\hline La Guajira & 31.120 & 8,1 & 29.474 & 8,1 & 26.779 & 7,8 & 26.746 & 8,1 & 23.005 & 7,3 \\
\hline Magdalena & 52.848 & 13,7 & 47.697 & 13,2 & 51.988 & 15,2 & 52.265 & 15,9 & 42.641 & 13,6 \\
\hline San Andrés & 2.165 & 0,6 & 2.001 & 0,6 & 1.988 & 0,6 & 2.279 & 0,7 & 1.735 & 0,6 \\
\hline Sucre & 27.110 & 7,0 & 22.759 & 6,3 & 22.622 & 6,6 & 22.497 & 6,8 & 21.128 & 6,7 \\
\hline Total & 384.659 & 100,0 & 362.127 & 100,0 & 341.917 & 100,0 & 329.479 & 100,0 & 313.911 & 100,0 \\
\hline \multicolumn{11}{|c|}{ Mujeres } \\
\hline \multirow{2}{*}{$\begin{array}{c}\text { Departa- } \\
\text { mento }\end{array}$} & \multicolumn{2}{|c|}{2004} & \multicolumn{2}{|c|}{2005} & \multicolumn{2}{|c|}{2006} & \multicolumn{2}{|c|}{2007} & \multicolumn{2}{|c|}{2008} \\
\hline & APVP & Part. \% & APVP & Part. \% & APVP & Part. \% & APVP & Part. \% & APVP & Part. \% \\
\hline Atlántico & 57.017 & 26,2 & 54.502 & 25,0 & 51.750 & 23,8 & 45.491 & 20,9 & 41.879 & 19,2 \\
\hline Bolívar & 39.090 & 17,9 & 42.852 & 19,7 & 35.048 & 16,1 & 28.614 & 13,1 & 33.331 & 15,3 \\
\hline
\end{tabular}


Andrés Castaño Zuluagua • Juan Correa Reyes • Luis Alvis Estrada • Nelson Alvis Guzmán

\begin{tabular}{|l|c|c|c|c|c|c|c|c|c|c|}
\hline \multicolumn{10}{|c|}{ Mujeres } \\
\hline \multirow{2}{*}{ Departamento } & \multicolumn{2}{|c|}{2004} & \multicolumn{2}{|c|}{2005} & \multicolumn{2}{c|}{2006} & \multicolumn{2}{|c|}{2007} & \multicolumn{2}{c|}{2008} \\
\cline { 2 - 13 } & APVP & Part. \% & APVP & Part. \% & APVP & Part. \% & APVP & Part. \% & APVP & Part. \% \\
\hline Cesar & 24.578 & 11,3 & 22.198 & 10,2 & 23.489 & 10,8 & 24.121 & 11,1 & 28.083 & 12,9 \\
\hline Córdoba & 35.719 & 16,4 & 30.850 & 14,2 & 28.935 & 13,3 & 29.120 & 13,4 & 28.095 & 12,9 \\
\hline La Guajira & 14.108 & 6,5 & 17.704 & 8,1 & 16.691 & 7,7 & 17.096 & 7,8 & 15.190 & 7,0 \\
\hline Magdalena & 31.351 & 14,4 & 29.049 & 13,3 & 28.074 & 12,9 & 31.054 & 14,3 & 27.096 & 12,4 \\
\hline San Andrés & 1.215 & 0,6 & 1.293 & 0,6 & 1.091 & 0,5 & 1.181 & 0,5 & 734 & 0,3 \\
\hline Sucre & 14.776 & 6,8 & 14.228 & 6,5 & 17.423 & 8,0 & 15.867 & 7,3 & 13.467 & 6,2 \\
\hline Total & 217.855 & 100,0 & 212.677 & 100,0 & 202.500 & 100,0 & 192.544 & 100,0 & 187.873 & 100,0 \\
\hline
\end{tabular}

Fuente: elaboración de los autores con base en DANE - Estadísticas vitales

Los resultados de las estimaciones al utilizar la metodología de los años potenciales de vida perdidos en el ámbito regional (tabla 2), muestran que los APVP totales (suma de hombres y mujeres) tienen clara tendencia a disminuir en el tiempo. Pasan de 602514 años perdidos en 2004 a 498652 en 2008, esto equivale a un disminución del 20,8 \% entre el 2004 y el 2008. El comportamiento de los APVP, obedece a que el impacto de la mortalidad en los menores de un año ha disminuido. Para efectos de esta metodología se pondera de una mayor manera a las defunciones de menor edad por lo cual cuando estas experimentan una diminución, el valor de los años potenciales de vida perdidos tiende a hacerlo también.

En la esfera departamental, Atlántico, seguido de Bolívar, Córdoba, Magdalena y Cesar son los que mayor cantidad de APVP generan. Sucre, La Guajira y San Andrés tienen los menores aportes. Aunque Atlántico y Bolívar sean los departamentos con mayor APVP, el comportamiento o la tendencia de estos son diferenciadas. Atlántico, en el año 2004, tenía 153936 APVP, mientras que para el año 2008 los APVP fueron de 112 715; esto equivale a una disminución del 26,7 \%. El caso de Atlántico es único en la región, ya que fue el único en donde, año a año, entre el 2004 y el 2008, sus APVP disminuyeron de manera consistente; este fenómeno es explicado, en gran parte, por la disminución que ha experimentado Atlántico en los índices de mortalidad en los más jóvenes, donde el número de muertes en los menores de un año ha disminuido en $16,3 \%$, y en la edad que concentra la mayor cantidad de defunciones por causa externa, que es el grupo de 15 a 44 años, la disminución ha sido aún más dinámica con 21,7% (Anexo 2). 
Valoración económica de la mortalidad en la región Caribe de Colombia, 2004-2008

Bolívar, por su parte, muestra una tendencia inestable: creciente, para unos años, decreciente, para otros. En el año 2004, Bolívar tenía 105719 APVP; para el año 2005 habían subido a 112 010, para decrecer, de manera sólida, entre 2006-2007 (de 97331 a 81075 APVP); en 2008, los APVP en Bolívar vuelven a la cifra de los 90 mil $(90$ 804). La inestabilidad de los APVP no es una constante solo para Bolívar: existen otros departamentos con la misma conducta. Cesar y Córdoba, comienzan el período de estudio con 76.661 y 91.505 APVP, respectivamente, luego en los años siguientes comienzan a disminuir, para luego, retomar en el año 2008 niveles de APVP muy parecidos a los del año inicial, en el caso de Cesar (72.862), y niveles aun bajos comparados con los del comienzo del período para el caso de Córdoba (80.409). En los casos de Bolívar y Cesar, la explicación de este fenómeno está relacionada con la proliferación de la mortalidad en las edades más avanzadas ( $>45$ años). Para el caso de Córdoba, la explicación pasa más por el comportamiento de la mortalidad en el grupo de edad más productivo (15 a 44 años). Entre 2004 y 2006, dicho grupo de edad exhibió una tendencia decreciente, pero a partir de 2007 los fenómenos de mortalidad se expandieron en cerca de 17 \%, jalonando el comportamiento de los APVP.

Por otra parte, para todos los años de estudio y en cada departamento del Caribe, el sexo masculino genera mayor número de APVP que el femenino, lo que indica una tendencia a la mortalidad temprana más marcada en hombres que en mujeres (tabla 2).

En lo que concierne a la valoración de los APVP en la región Caribe colombiana (tabla 3), las pérdidas originadas por la mortalidad evitable en todo el periodo 2004 2008 equivalen a 15,09 billones de pesos aproximadamente (a precios del 2008). El año 2004, es el año en el que más pérdidas se presentan en la esfera regional (3,23 billones de pesos), mientras que el 2008 es el de menos pérdidas (2,76 billones). El valor de los APVP en dólares para el período 2004-2008 fue de 8,474 millones de dólares y representa en promedio el 5,2 \% del PIB en el ámbito regional. La tendencia que muestra el valor económico de los APVP en el periodo de estudio es decreciente.

En el ámbito departamental, si bien se muestra una tendencia decreciente tanto en el valor económico de las vidas como en su participación regional, departamentos como Bolívar, Cesar y Córdoba han aumentado su participación dentro de la valoración regional, en detrimento de departamentos como Atlántico que muestra patrones claros de disminución de las muertes entre 2004 y 2008 en los grupos de edad de menores de 1 año, y de 15 a 44 años.

Semestre Económico, volumen 16, №. 33, pp. 155-180 • ISSN 0120-6346, enero-junio de 2013, Medellín, Colombia 
Andrés Castaño Zuluagua • Juan Correa Reyes • Luis Alvis Estrada • Nelson Alvis Guzmán

Tabla 3. Estimaciones del valor económico de los APVP de los departamentos de la región Caribe en el período 2004-2008 y comparación con el PIB regional (miles de millones de pesos de 2008)

\begin{tabular}{|c|c|c|c|c|c|c|c|c|}
\hline Depart. & Año & $\begin{array}{c}\text { Valor } \\
\text { económico } \\
\text { APVP }\end{array}$ & $\begin{array}{c}\text { Part. } \\
\text { Regional } \\
\text { (\%) }\end{array}$ & $\begin{array}{c}\text { Variación } \\
\text { valor } \\
\text { APVP }\end{array}$ & PIB & $\begin{array}{c}\text { Part. } \\
\text { Regional } \\
\text { (\%) }\end{array}$ & $\begin{array}{c}\text { Variación } \\
\text { PIB }\end{array}$ & \begin{tabular}{|c|} 
Partic. \\
Valor \\
APVP en el \\
PIB (\%)
\end{tabular} \\
\hline \multirow{5}{*}{ Atlántico } & 2004 & 824,5 & 25,55 & & 9998,8 & 26,44 & & 8,25 \\
\hline & 2005 & 790,9 & 25,28 & $-4,07$ & 11633,1 & 26,56 & 16,3 & 6,80 \\
\hline & 2006 & 781,4 & 25,67 & $-1,21$ & 13945,7 & 26,55 & 19,9 & 5,60 \\
\hline & 2007 & 695,5 & 23,72 & $-10,99$ & 17338,3 & 27,35 & 24,3 & 4,01 \\
\hline & 2008 & 606,9 & 21,98 & $-12,75$ & 20091,0 & 26,66 & 15,9 & 3,02 \\
\hline \multirow{5}{*}{ Bolívar } & 2004 & 566,2 & 17,55 & & 9844,8 & 26,04 & & 5,75 \\
\hline & 2005 & 609,7 & 19,49 & 7,68 & 11146,2 & 25,45 & 13,2 & 5,47 \\
\hline & 2006 & 542,3 & 17,82 & $-11,05$ & 13539,7 & 25,78 & 21,5 & 4,01 \\
\hline & 2007 & 454,3 & 15,50 & $-16,22$ & 16293,5 & 25,70 & 20,3 & 2,79 \\
\hline & 2008 & 502,9 & 18,21 & 10,68 & 18875,0 & 25,05 & 15,8 & 2,66 \\
\hline \multirow{5}{*}{ Cesar } & 2004 & 410,6 & 12,72 & & 4306,4 & 11,39 & & 9,53 \\
\hline & 2005 & 374,3 & 11,96 & $-8,85$ & 5230,4 & 11,94 & 21,5 & 7,16 \\
\hline & 2006 & 357,0 & 11,73 & $-4,61$ & 6198,3 & 11,80 & 18,5 & 5,76 \\
\hline & 2007 & 389,2 & 13,28 & 9,02 & 6989,6 & 11,03 & 12,8 & 5,57 \\
\hline & 2008 & 403,5 & 14,61 & 3,68 & 9738,0 & 12,92 & 39,3 & 4,14 \\
\hline \multirow{5}{*}{ Córdoba } & 2004 & 490,1 & 15,19 & & 5389,2 & 14,25 & & 9,09 \\
\hline & 2005 & 460,1 & 14,71 & $-6,12$ & 5995,7 & 13,69 & 11,3 & 7,67 \\
\hline & 2006 & 434,0 & 14,26 & $-5,68$ & 7309,8 & 13,92 & 21,9 & 5,94 \\
\hline & 2007 & 445,7 & 15,20 & 2,69 & 9442,3 & 14,89 & 29,2 & 4,72 \\
\hline & 2008 & 445,3 & 16,13 & $-0,09$ & 9003,0 & 11,95 & $-4,7$ & 4,95 \\
\hline \multirow{5}{*}{ La Guajira } & 2004 & 242,2 & 7,51 & & 2714,3 & 7,18 & & 8,92 \\
\hline & 2005 & 256,8 & 8,21 & 6,01 & 3300,2 & 7,53 & 21,6 & 7,78 \\
\hline & 2006 & 242,2 & 7,96 & $-5,69$ & 3887,3 & 7,40 & 17,8 & 6,23 \\
\hline & 2007 & 245,7 & 8,38 & 1,43 & 4227,5 & 6,67 & 8,8 & 5,81 \\
\hline & 2008 & 211,5 & 7,66 & $-13,90$ & 6598,0 & 8,76 & 56,1 & 3,21 \\
\hline
\end{tabular}


Valoración económica de la mortalidad en la región Caribe de Colombia, 2004-2008

\begin{tabular}{|c|c|c|c|c|c|c|c|c|}
\hline Depart. & Año & $\begin{array}{c}\text { Valor } \\
\text { económico } \\
\text { APVP }\end{array}$ & $\begin{array}{c}\text { Part. } \\
\text { Regional } \\
(\%)\end{array}$ & $\begin{array}{c}\text { Variación } \\
\text { valor } \\
\text { APVP }\end{array}$ & PIB & $\begin{array}{c}\text { Part. } \\
\text { Regional } \\
(\%)\end{array}$ & $\begin{array}{c}\text { Variación } \\
\text { PIB }\end{array}$ & $\begin{array}{c}\text { Partic. } \\
\text { Valor } \\
\text { APVP en el } \\
\text { PIB (\%) }\end{array}$ \\
\hline \multirow{5}{*}{ Magdalena } & 2004 & 451,0 & 13,97 & & 3282,1 & 8,68 & & 13,74 \\
\hline & 2005 & 417,8 & 13,35 & $-7,37$ & 3905,7 & 8,92 & 19,0 & 10,70 \\
\hline & 2006 & 446,1 & 14,66 & 6,79 & 4483,1 & 8,54 & 14,8 & 9,95 \\
\hline & 2007 & 466,9 & 15,93 & 4,66 & 5391,2 & 8,50 & 20,3 & 8,66 \\
\hline & 2008 & 386,2 & 13,99 & $-17,28$ & 6639,0 & 8,81 & 23,1 & 5,82 \\
\hline \multirow{5}{*}{ San Andrés } & 2004 & 18,1 & 0,56 & & 458,8 & 1,21 & & 3,95 \\
\hline & 2005 & 17,9 & 0,57 & $-0,95$ & 504,6 & 1,15 & 10,0 & 3,55 \\
\hline & 2006 & 17,2 & 0,56 & $-4,31$ & 591,4 & 1,13 & 17,2 & 2,90 \\
\hline & 2007 & 19,4 & 0,66 & 13,02 & 728,1 & 1,15 & 23,1 & 2,66 \\
\hline & 2008 & 13,7 & 0,50 & $-29,49$ & 847,0 & 1,12 & 16,3 & 1,61 \\
\hline \multirow{5}{*}{ Sucre } & 2004 & 224,3 & 6,95 & & 1816,7 & 4,80 & & 12,35 \\
\hline & 2005 & 201,3 & 6,43 & $-10,25$ & 2082,4 & 4,75 & 14,6 & 9,67 \\
\hline & 2006 & 223,1 & 7,33 & 10,82 & 2569,3 & 4,89 & 23,4 & 8,68 \\
\hline & 2007 & 215,0 & 7,33 & $-3,65$ & 2984,0 & 4,71 & 16,1 & 7,20 \\
\hline & 2008 & 191,6 & 6,94 & $-10,89$ & 3571,0 & 4,74 & 19,7 & 5,36 \\
\hline \multirow{5}{*}{ Región } & 2004 & $3.227,1$ & 100,0 & & 37811,3 & 100,0 & & 8,53 \\
\hline & 2005 & $3.128,9$ & 100,0 & $-3,04$ & 43798,3 & 100,0 & 15,8 & 7,14 \\
\hline & 2006 & $3.043,3$ & 100,0 & $-2,74$ & 52524,6 & 100,0 & 19,9 & 5,79 \\
\hline & 2007 & $2.931,7$ & 100,0 & $-3,67$ & 63394,6 & 100,0 & 20,7 & 4,62 \\
\hline & 2008 & $2.761,5$ & 100,0 & $-5,80$ & 75362,0 & 100,0 & 18,9 & 3,66 \\
\hline
\end{tabular}

Fuente: elaboración de los autores con base en DANE - Estadísticas Vitales.

A pesar de que la tendencia que muestra la participación del valor económico de las muertes dentro del PIB regional es decreciente, este comportamiento no debe interpretarse como algo positivo; más bien se debe aclarar que obedece a que el PIB regional ha crecido a una tasa promedio para el período de $12,5 \%$, mientras que el valor económico de las vidas perdidas lo hace a una tasa promedio para el periodo de $-3,5 \%$. Lo anterior indica que a pesar de que se ha logrado disminuir las cifras de mortalidad, y con ello los años potenciales de vida perdidos de algunos 
grupos de edad neurálgicos, como el de menores de 1 año, y el de 15 a 44 años, se debe tener cuidado con las particularidades o patrones de mortalidad de cada departamento.

La mortalidad es un fenómeno con características propias de cada unidad territorial. En general, para la región Caribe se ha logrado disminuir la mortalidad en los menores de un año y en el grupo de 15 a 44 años. El primer grupo normalmente es para el que se obtienen, en promedio, mayor cantidad de años perdidos (controlando por número de defunciones), por lo cual los resultados de las estimaciones son coherentes. Por su parte, es muy representativo y positivo para la región que la mortalidad en un grupo de edad neurálgico para el mercado laboral y para el consumo de bienes y servicios, como el de 15 a 44 años, esté mostrando una tendencia decreciente.

\section{CONCLUSIONES}

Usando las estadísticas vitales del DANE entre 2004 y 2008, este documento aplica la metodología del capital humano de valoración de la vida, para estimar el valor económico de los años de vida perdidos en los departamentos de la región Caribe. Este método ha sido anteriormente utilizado por Alvis y Alvis (2009), Alvis y de la Hoz (2004), Alvis y otros (2009), entre otros. En este trabajo se alcanza una dimensión de análisis hasta ahora ausente en la bibliografía: se estima el valor económico para una región y sus departamentos, teniendo en cuenta aspectos como el sexo y grupos de edad por departamento. En estudios anteriores como el de Alvis y otros (2009), el foco se centraba en la valoración económica para una enfermedad; para un grupo de enfermedades, en el caso de Alvis y de la Hoz (2004), o para un municipio, como Alvis y Alvis (2009).

Si bien, este trabajo arroja conclusiones que no son comparables con estudios que aplican la misma metodología, la riqueza o el aporte adicional de estudiar una región de manera íntegra es que permite entender de mejor manera la dinámica de la mortalidad en el área departamental y su relación con los otros entes territoriales; además, que sirve como punto de comparación en la medida en que se encuentre evidencia de experiencias de control de la mortalidad positivas en unos departamentos con respecto a otros en donde las tendencias de mortalidad de algún grupo de causas son desfavorables.

Los resultados, en términos desagregados, muestran que en algunos departamentos las defunciones crecen a una mayor tasa promedio que en otros. Ejemplos claros de esta situación son La Guajira y Sucre que, al contrario de Bolívar, Atlántico y Magdalena, presentan las tasas de crecimiento promedio de las defunciones más 
Valoración económica de la mortalidad en la región Caribe de Colombia, 2004-2008

altas de la región. La situación en Atlántico merece una mirada más detallada de sus tendencias de mortalidad, ya que es dentro de la región el único departamento con un comportamiento casi invariable en términos de defunciones de 2004 a 2008; asimismo, muestra cifras de mortalidad menores en los grupos de edad de menores de 1 año, y de 15 a 44 años (Anexo 2), que son en términos de valor económico, de mayor importancia económica relativa. Lo anterior ha permitido que departamentos como Bolívar y Cesar ganen participación relativa.

Dos rasgos muy interesantes distinguen la mortalidad en el área regional; en primera instancia, los grupos de mayor edad (45 en adelante) sufren aumentos importantes y sostenidos en el tiempo en cuanto a su número de defunciones; esto podría obedecer a un descuido en las políticas en materia de salud que buscan proteger a los adultos para que puedan tener una vejez tranquila y así en muchos casos poder dedicar los últimos años de su vida de manera íntegra a su familia después de entregarle los más productivos al mercado laboral. El segundo punto de discusión importante es que los grupos de menor edad (menores de 45 años) han sufrido disminuciones importantes en materia de eventos de mortalidad; esto puede ser benéfico en el largo plazo dado que la gente que se encuentra en esta edad tiende a ser más productiva y aportar mayor cantidad de consumo de bienes y servicios, por lo cual su impacto dentro de la producción nacional es importante.

Más allá de los resultados que en el área regional sugieren que las políticas sanitarias están teniendo efecto positivo sobre la disminución de la mortalidad, es imprescindible mencionar que por departamento y controlando por causas agrupadas esta afirmación puede arrojar interpretaciones erróneas y recomendaciones de política bastante lejanas a la realidad de cada departamento. Al tener en cuenta las causas agrupadas de defunción por departamento, las políticas deben estar orientadas hacia aquellas defunciones o grupo de defunciones que muestran indicios de aumento para el período de estudio.

En Atlántico, Bolívar, Cesar y Magdalena, se debe prestar particular atención al comportamiento de las defunciones provocadas por neoplastias (tumores), que muestran una tendencia de crecimiento preocupante. En Córdoba, el énfasis se debe colocar en la mortalidad por causas externas, principalmente en materia de homicidios. Córdoba, ha sido después del proceso de desmovilización armada, uno de los departamentos con mayor aumento en los homicidios, no solo por la conveniencia que ven los grupos al margen de la ley, dada su posición estratégica entre la costa caribe y el interior del país, sino también por ser uno de los departamentos con una estructura productiva (mayormente agrícola) que los favorece en el desarrollo de sus actividades delictivas. 
Finalmente, es imprescindible mencionar que, a pesar de que la base de datos obtenida a partir de las estadísticas vitales del DANE es muy valiosa, no cuenta con el nivel de desagregación necesario para llegar a reflexiones concluyentes sobre las tendencias de mortalidad en los departamentos de la región Caribe; asimismo, es importante hacer notar que en este trabajo no se asume ninguna posición respecto al tratamiento del subregistro, el cual es un problema inherente a cualquier base de datos que lleve registros de mortalidad.

\section{BIBLIOGRAFÍA}

Alvis, N. y Alvis, L. (2009). Costos económicos de la mortalidad evitable en Cartagena, Colombia, 2000-2005. En: Revista de Salud Pública. Vol. 11, N. ${ }^{\circ}$ 6, pp. 970-978.

Alvis, N.; Correa, J.; López, C. y Pattigno, G. (2009) Impacto económico de la mortalidad por SIDA en Colombia 1997-2001. En: Revista Panorama Económico. N. 15, pp. 145-169.

Alvis, N. y De La Hoz, F. (2004). Tendencias de la mortalidad por enfermedades infecciosas en Cartagena de Indias, Colombia, 1995-2000. En: Revista de Salud Pública. Vol. 6, N. 3, pp. 235-252.

Arriaga, E. (1984). Measuring and explaining the change of life expectancies. En: Demography. Vol. 21, N. ${ }^{\circ} 1$, pp. 83-96.

Arriaga E. (1996). Los años de vida perdidos: su utilización para medir el nivel y el cambio de la mortalidad. En: Notas de Población. Vol. 24, N. ${ }^{\circ}$ 63, pp. 7-38.

Bertone, C.; Andrada, M. y Peranovich, A. (2008). Análisis comparativo de la mortalidad por grandes grupos de causas de muerte en el estado de Piaú (Brasil) y la provincia de Chaco (Argentina) en el período 2000-2005. III Congreso de la Asociación Latinoamericana de Población, ALAP. Córdoba -Argentina.

Bocco, M. (1996). La relación entre los años de vida perdidos y la esperanza de vida: Aplicaciones para el análisis de la mortalidad. En: Notas de Población. Vol. 24, N. 63, pp. 39-60.

Boleda M, Arriaga E. (2000). América Latina: mortalidad por accidentes y por violencia contra las personas. En: Notas de Población. Vol. 28, N. ${ }^{\circ} 70$, pp. 87-119. Disponible en: http://www. eclac.org/publicaciones/xml/6/6776/lcg21003.pdf .

Cruz, Salvador y Muñoz, María (2005). Some considerations on the social discount rate. En: Environmental Science and Policy. N. ${ }^{\circ}$ 8, pp. 343-355.

Dempsey, M. (1947). Decline in tuberculosis: The death rate fails to tell the entire story. En: Am Rev Tubercul. Vol. 86, pp. 157-64.

Dickinson F. y Welker E. (1948). What is the leading cause of death? Two new measures. En: Bureau of Medical Economic Research. American Medical Association. Bulletin 64.

Haenszel W. (1950). A standardized rate for mortality defined in units of lost years of life. En: Am J Public Health. Vol 40, pp. 17-26. 
Valoración económica de la mortalidad en la región Caribe de Colombia, 2004-2008

Farr, W. (1853). The Income and Property Tax. En: Journal of the Statistical Society of London. Vol. 16, pp. 1 44.

Landry, A. (1934). Le révolution démographique - Études et essais sur les problèmes de la population. INED-Presses Universitaires de France.

López, E., Arce, P. (2008). Efectos de las causas de mortalidad adulta en la esperanza de vida, entre 1985 y 1999, según regiones colombianas. En: Biomédica, Vol. 28, N. 3, pp. 414-422.

OPS -Organización Panamericana de la Salud- (1995). Clasificación Estadística Internacional de Enfermedades y Problemas Relacionados con la Salud. Décima Revisión. Vols. 1-3. Washington, DC.

Pérez, M., Santiago M.; Cerdeira, S., Alonso, B.; Malvar, A. Y Hervada, X. (2009) Mortalidad y años de esperanza de vida perdidos a causa del tabaquismo en personas mayores de 35 años en Galicia en el período 2001 - 2006. En: Revista Española de Salud pública. Vol. 83, N. ${ }^{\circ}$, pp. 557-565.

Petty, W. (1960). Political Arithmetick. En: Archive for the History of Economic Thought. MacMater University, Canadá.

Smith A. (1776). An inquiry into the nature and causes of the wealth of nations. London. Edited by The electric Book Company Ltd, p. 1227.

Toro, M.; García, O.; Sanchez, J. y Moreno R. (2007). Años de vida potencialmente perdidos por la población de municipio de Itagüí. CES, Facultad de Medicina. División de Salud Pública, 49 pp. 
Andrés Castaño Zuluagua • Juan Correa Reyes • Luis Alvis Estrada • Nelson Alvis Guzmán

\section{ANEXOS}

Anexo 1. Mortalidad por causas agrupadas en los departamentos de la Región Caribe, 2004-20084.

\begin{tabular}{|c|c|c|c|c|c|c|c|c|c|c|c|c|c|c|c|c|c|c|c|}
\hline & \multirow{2}{*}{ Causa } & \multicolumn{2}{|c|}{ Atlántico } & \multicolumn{2}{|c|}{ Bolivar } & \multicolumn{2}{|c|}{ Cesar } & \multicolumn{2}{|c|}{ Córdoba } & \multicolumn{2}{|c|}{ La Guajira } & \multicolumn{2}{|c|}{ Magdalena } & \multicolumn{2}{|c|}{ San Andrés } & \multicolumn{2}{|c|}{ Sucre } & \multicolumn{2}{|c|}{ Región } \\
\hline & & M & $\%$ & M & $\%$ & M & $\%$ & M & $\%$ & M & $\%$ & M & $\%$ & M & $\%$ & M & $\%$ & M & $\%$ \\
\hline \multirow{7}{*}{$\begin{array}{l}\text { 옹 } \\
\stackrel{1}{ }\end{array}$} & 1 & 886 & 10,0 & 568 & 10,4 & 286 & 8,9 & 473 & 10,7 & 153 & 9,9 & 322 & 8,3 & 8 & 3,9 & 211 & 8,6 & 1188 & 9,5 \\
\hline & 2 & 1414 & 16,0 & 882 & 16,1 & 429 & 13,4 & 652 & 14,7 & 173 & 11,1 & 525 & 13,5 & 32 & 15,5 & 372 & 15,2 & 2232 & 17,9 \\
\hline & 3 & 2949 & 33,3 & 1755 & 32,0 & 824 & 25,7 & 1468 & 33,1 & 303 & 19,5 & 1220 & 31,3 & 70 & 33,8 & 908 & 37,1 & 4564 & 36,5 \\
\hline & 4 & 365 & 4,1 & 297 & 5,4 & 174 & 5,4 & 283 & 6,4 & 82 & 5,3 & 227 & 5,8 & 10 & 4,8 & 61 & 2,5 & 635 & 5,1 \\
\hline & 5 & 1196 & 13,5 & 694 & 12,7 & 859 & 26,8 & 582 & 13,1 & 549 & 35,4 & 736 & 18,9 & 35 & 16,9 & 401 & 16,4 & 580 & 4,6 \\
\hline & 6 & 2043 & 23,1 & 1280 & 23,4 & 639 & 19,9 & 979 & 22,1 & 292 & 18,8 & 866 & 22,2 & 52 & 25,1 & 496 & 20,3 & 3305 & 26,4 \\
\hline & Total & 8853 & 100 & 5476 & 100 & 3211 & 100 & 4437 & 100 & 1552 & 100 & 3896 & 100 & 207 & 100 & 2449 & 100 & 12504 & 100 \\
\hline \multirow{7}{*}{ 오 } & 1 & 928 & 10,6 & 605 & 10,4 & 257 & 8,1 & 376 & 8,6 & 186 & 11,1 & 308 & 7,7 & 14 & 6,3 & 194 & 8,2 & 1218 & 9,4 \\
\hline & 2 & 1435 & 16,4 & 889 & 15,3 & 434 & 13,8 & 646 & 14,8 & 202 & 12,0 & 631 & 15,9 & 48 & 21,5 & 372 & 15,8 & 2358 & 18,2 \\
\hline & 3 & 2729 & 31,2 & 1777 & 30,6 & 796 & 25,2 & 1466 & 33,6 & 358 & 21,3 & 1259 & 31,6 & 63 & 28,3 & 859 & 36,4 & 4483 & 34,7 \\
\hline & 4 & 385 & 4,4 & 295 & 5,1 & 178 & 5,6 & 300 & 6,9 & 145 & 8,6 & 224 & 5,6 & 9 & 4,0 & 67 & 2,8 & 673 & 5,2 \\
\hline & 5 & 1099 & 12,6 & 878 & 15,1 & 791 & 25,1 & 551 & 12,6 & 461 & 27,4 & 610 & 15,3 & 34 & 15,2 & 304 & 12,9 & 675 & 5,2 \\
\hline & 6 & 2177 & 24,9 & 1372 & 23,6 & 699 & 22,2 & 1028 & 23,5 & 329 & 19,6 & 946 & 23,8 & 55 & 24,7 & 561 & 23,8 & 3530 & 27,3 \\
\hline & Total & 8753 & 100 & 5816 & 100 & 3155 & 100 & 4367 & 100 & 1681 & 100 & 3978 & 100 & 223 & 100 & 2357 & 100 & 12937 & 100 \\
\hline \multirow{7}{*}{$\begin{array}{l}\text { 용 } \\
\text { ᄋ }\end{array}$} & 1 & 854 & 9,4 & 614 & 10,5 & 299 & 9,7 & 372 & 8,5 & 206 & 9,6 & 320 & 10,0 & 14 & 1,8 & 184 & 5,6 & 1189 & 9,0 \\
\hline & 2 & 1489 & 16,4 & 909 & 15,6 & 463 & 15,0 & 642 & 14,7 & 195 & 9,1 & 644 & 20,0 & 43 & 5,7 & 388 & 11,8 & 2397 & 18,1 \\
\hline & 3 & 2859 & 31,4 & 1863 & 31,9 & 845 & 27,4 & 1500 & 34,3 & 328 & 15,3 & 1278 & 39,8 & 70 & 9,2 & 937 & 28,4 & 4617 & 34,8 \\
\hline & 4 & 397 & 4,4 & 208 & 3,6 & 194 & 6,3 & 238 & 5,4 & 90 & 4,2 & 256 & 8,0 & 10 & 1,3 & 91 & 2,8 & 606 & 4,6 \\
\hline & 5 & 1135 & 12,5 & 853 & 14,6 & 687 & 22,3 & 560 & 12,8 & 369 & 17,2 & 668 & 20,8 & 27 & 3,6 & 331 & 10,0 & 728 & 5,5 \\
\hline & 6 & 2365 & 26,0 & 1388 & 23,8 & 592 & 19,2 & 1067 & 24,4 & 962 & 44,7 & 49 & 1,5 & 595 & 78,4 & 1364 & 41,4 & 3722 & 28,1 \\
\hline & Total & 9099 & 100 & 5835 & 100 & 3080 & 100 & 4379 & 100 & 2150 & 100 & 3215 & 100 & 759 & 100 & 3295 & 100 & 13259 & 100 \\
\hline
\end{tabular}

* Los números que denotan las causas corresponden a la siguiente relación: enfermedades transmisibles (1), neoplastias (2), enfermedades del sistema circulatorio (3), afecciones perinatales (4), causas externas (5), demás causas (6). 
Valoración económica de la mortalidad en la región Caribe de Colombia, 2004-2008

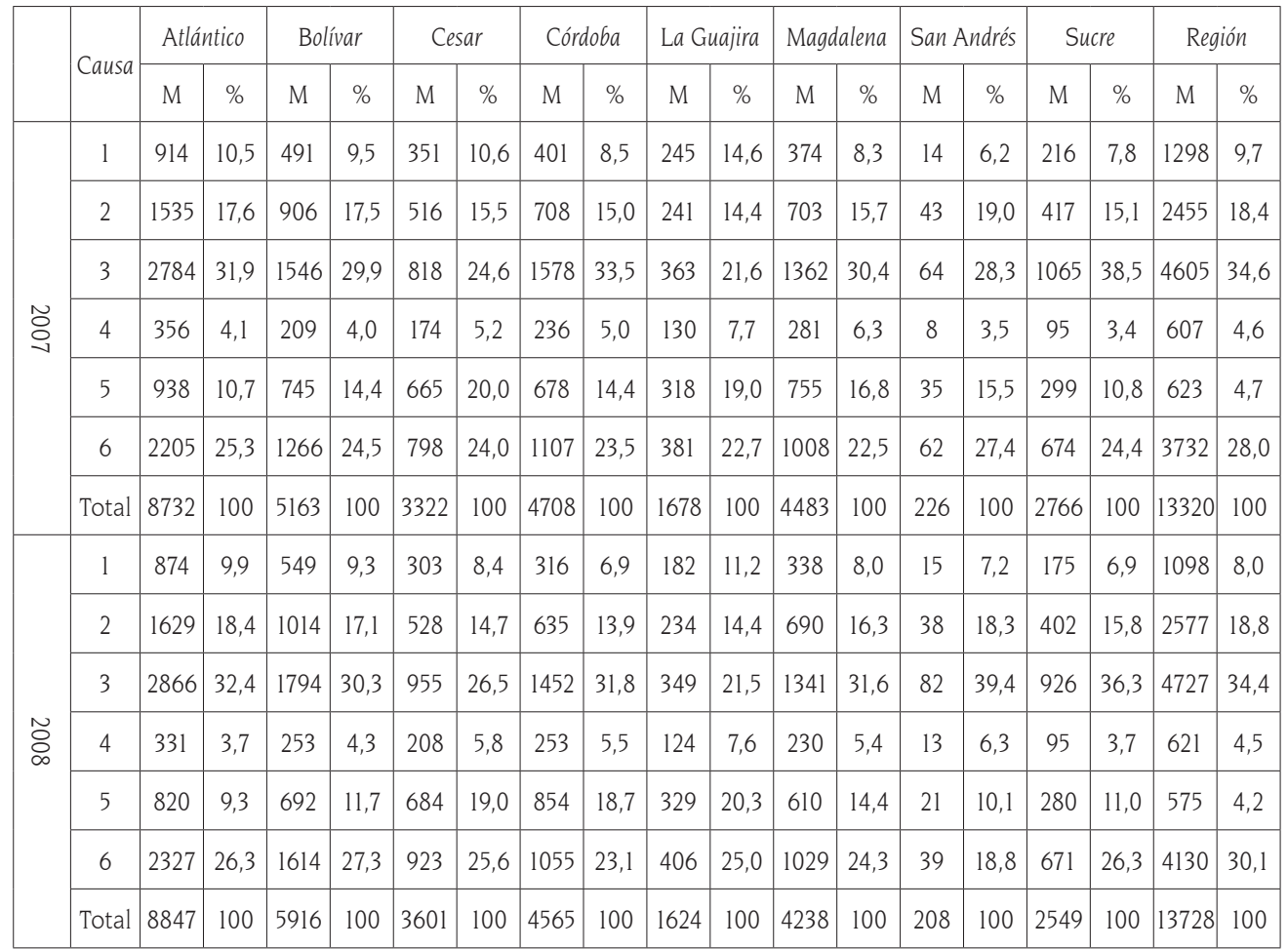

Fuente: DANE - Estadísticas Vitales, Cuentas Departamentales. Cálculos de los autores.

Anexo 2. Mortalidad por grupo de edad en los departamentos de la Región Caribe,

2004-2008

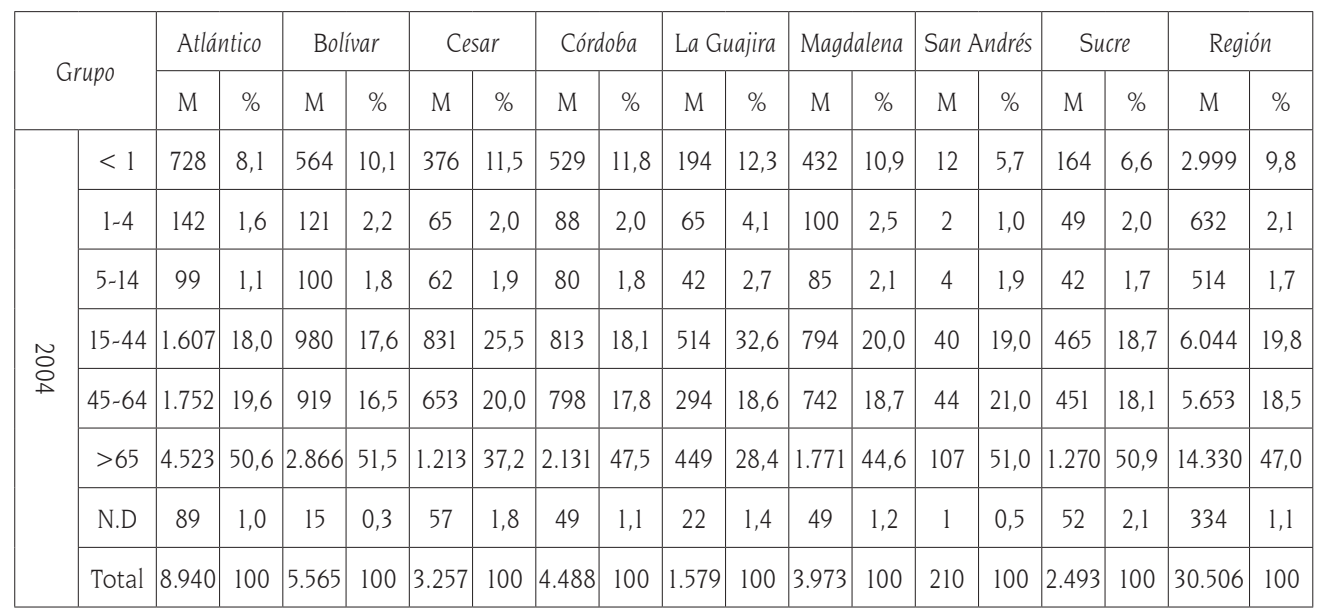


Andrés Castaño Zuluagua • Juan Correa Reyes • Luis Alvis Estrada • Nelson Alvis Guzmán

\begin{tabular}{|c|c|c|c|c|c|c|c|c|c|c|c|c|c|c|c|c|c|c|c|}
\hline \multirow{2}{*}{\multicolumn{2}{|c|}{ Grupo }} & \multicolumn{2}{|c|}{ Atlántico } & \multicolumn{2}{|c|}{ Bolivar } & \multicolumn{2}{|c|}{ Cesar } & \multicolumn{2}{|c|}{ Córdoba } & \multicolumn{2}{|c|}{ La Guajira } & \multicolumn{2}{|c|}{ Magdalena } & \multicolumn{2}{|c|}{ San Andrés } & \multicolumn{2}{|c|}{ Sucre } & \multicolumn{2}{|c|}{ Región } \\
\hline & & M & $\%$ & M & $\%$ & M & $\%$ & M & $\%$ & M & $\%$ & M & $\%$ & M & $\%$ & M & $\%$ & M & $\%$ \\
\hline \multirow{8}{*}{8} & $<1$ & 750 & 8,5 & 616 & 10,4 & 336 & 10,5 & 536 & 12,0 & 277 & 16,4 & 429 & 10,6 & 14 & 6,3 & 175 & 7,2 & 3.133 & 10,2 \\
\hline & $1-4$ & 134 & 1,5 & 125 & 2,1 & 66 & 2,1 & 75 & 1,7 & 52 & 3,1 & 75 & 1,9 & 0 & 0,0 & 34 & 1,4 & 561 & 1,8 \\
\hline & $5-14$ & 113 & 1,3 & 91 & 1,5 & 61 & 1,9 & 79 & 1,8 & 42 & 2,5 & 100 & 2,5 & 3 & 1,3 & 39 & 1,6 & 528 & 1,7 \\
\hline & $15-44$ & 1.478 & 16,7 & 1.106 & 18,7 & 777 & 24,3 & 716 & 16,1 & 473 & 27,9 & 705 & 17,4 & 40 & 17,9 & 381 & 15,6 & 5.676 & 18,4 \\
\hline & $45-64$ & 1.665 & 18,8 & 1.014 & 17,1 & 593 & 18,5 & 790 & 17,7 & 283 & 16,7 & 695 & 17,2 & 50 & 22,3 & 453 & 18,6 & 5.543 & 18,0 \\
\hline & $>65$ & 4.626 & 52,3 & 2.945 & 49,8 & 1.323 & 41,3 & 2.245 & 50,3 & 553 & 32,7 & 2.010 & 49,7 & 115 & 51,3 & 1.313 & 53,9 & 15.130 & 49,1 \\
\hline & N.D & 79 & 0,9 & 22 & 0,4 & 48 & 1,5 & 19 & 0,4 & 13 & 0,8 & 31 & 0,8 & 2 & 0,9 & 40 & 1,6 & 254 & 0,8 \\
\hline & Total & 8.845 & 100 & 5.919 & 100 & 3.204 & 100 & 4.460 & 100 & 1.693 & 100 & 4.045 & 100 & 224 & 100 & 2.435 & 100 & 30.825 & 100 \\
\hline \multirow{8}{*}{ 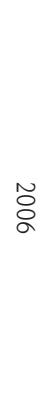 } & $<1$ & 746 & 8,1 & 471 & 7,9 & 367 & 11,8 & 438 & 9,8 & 259 & 16,3 & 477 & 11,4 & 15 & 7,0 & 210 & 8,2 & 2.983 & 9,5 \\
\hline & $1-4$ & 114 & 1,2 & 102 & 1,7 & 67 & 2,2 & 89 & 2,0 & 93 & 5,9 & 78 & 1,9 & 4 & 1,9 & 52 & 2,0 & 599 & 1,9 \\
\hline & $5-14$ & 138 & 1,5 & 103 & 1,7 & 66 & 2,1 & 92 & 2,1 & 40 & 2,5 & 89 & 2,1 & 25 & 11,6 & 47 & 1,8 & 600 & 1,9 \\
\hline & $15-44$ & 1.449 & 15,7 & 1.070 & 18,0 & 642 & 20,7 & 692 & 15,5 & 385 & 24,2 & 770 & 18,4 & 32 & 14,9 & 375 & 14,7 & 5.415 & 17,3 \\
\hline & 45-64 & 1.699 & 18,4 & 1.075 & 18,1 & 579 & 18,6 & 795 & 17,8 & 248 & 15,6 & 719 & 17,2 & 78 & 36,3 & 479 & 18,7 & 5.672 & 18,1 \\
\hline & $>65$ & 4.981 & 54,1 & 3.115 & 52,3 & 1.371 & 44,1 & 2.333 & 52,2 & 556 & 35,0 & 2.041 & 48,7 & 61 & 28,4 & 1.364 & 53,4 & 15.822 & 50,6 \\
\hline & N.D & 82 & 0,9 & 16 & 0,3 & 16 & 0,5 & 29 & 0,6 & 8 & 0,5 & 18 & 0,4 & 0 & 0,0 & 28 & 1,1 & 197 & 0,6 \\
\hline & Total & 9.209 & 100 & 5.952 & 100 & 3.108 & 100 & 4.466 & 100 & 1.588 & 100 & 4.192 & 100 & 215 & 100 & 2.555 & 100 & 31.288 & 100 \\
\hline \multirow{8}{*}{$\tilde{8}$} & $<1$ & 690 & 7,8 & 407 & 7,8 & 412 & 12,2 & 453 & 9,4 & 302 & 17,9 & 484 & 10,7 & 16 & 7,0 & 202 & 7,2 & 2.966 & 9,4 \\
\hline & $1-4$ & 89 & 1,0 & 65 & 1,2 & 81 & 2,4 & 75 & 1,6 & 74 & 4,4 & 92 & 2,0 & 4 & 1,8 & 35 & 1,3 & 515 & 1,6 \\
\hline & $5-14$ & 119 & 1,4 & 81 & 1,5 & 64 & 1,9 & 85 & 1,8 & 41 & 2,4 & 77 & 1,7 & 35 & 15,4 & 52 & 1,9 & 554 & 1,8 \\
\hline & $15-44$ & 1.320 & 15,0 & 944 & 18,0 & 731 & 21,7 & 815 & 16,9 & 353 & 20,9 & 880 & 19,4 & 29 & 12,8 & 397 & 14,2 & 5.469 & 17,4 \\
\hline & $45-64$ & 1.616 & 18,4 & 997 & 19,0 & 599 & 17,8 & 815 & 16,9 & 287 & 17,0 & 801 & 17,7 & 81 & 35,7 & 481 & 17,2 & 5.677 & 18,0 \\
\hline & $>65$ & 4.921 & 55,9 & 2.736 & 52,1 & 1.449 & 43,0 & 2.560 & 53,0 & 616 & 36,4 & 2.173 & 48,0 & 62 & 27,3 & 1.619 & 57,9 & 16.136 & 51,2 \\
\hline & N.D & 49 & 0,6 & 22 & 0,4 & 34 & 1,0 & 31 & 0,6 & 17 & 1,0 & 19 & 0,4 & 0 & 0,0 & 12 & 0,4 & 184 & 0,6 \\
\hline & Total & 8.804 & 100 & 5.249 & 100 & 3.367 & 100 & 4.834 & 100 & 1.690 & 100 & 4.526 & 100 & 227 & 100 & 2.798 & 100 & 31.501 & 100 \\
\hline \multirow{8}{*}{ ○ } & $<1$ & 626 & 7,0 & 516 & 8,5 & 402 & 10,9 & 455 & 9,7 & 247 & 15,0 & 410 & 9,5 & 18 & 8,6 & 206 & 8,0 & 2.880 & 9,0 \\
\hline & $1-4$ & 70 & 0,8 & 77 & 1,3 & 88 & 2,4 & 68 & 1,5 & 73 & 4,4 & 65 & 1,5 & 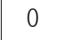 & 0,0 & 21 & 0,8 & 462 & 1,4 \\
\hline & $5-14$ & 96 & 1,1 & 109 & 1,8 & 69 & 1,9 & 96 & 2,1 & 39 & 2,4 & 68 & 1,6 & 2 & 1,0 & 38 & 1,5 & 517 & 1,6 \\
\hline & $15-44$ & 1.258 & 14,0 & 989 & 16,3 & 764 & 20,8 & 947 & 20,2 & 356 & 21,6 & 771 & 17,9 & 21 & 10,0 & 358 & 13,9 & 5.464 & 17,0 \\
\hline & 45-64 & 1.712 & 19,1 & 1.100 & 18,1 & 700 & 19,1 & 749 & 16,0 & 276 & 16,7 & 770 & 17,9 & 43 & 20,5 & 493 & 19,1 & 5.843 & 18,2 \\
\hline & $>65$ & 5.139 & 57,4 & 3.262 & 53,6 & 1.612 & 43,9 & 2.347 & 50,2 & 636 & 38,6 & 2.218 & 51,5 & 124 & 59,0 & 1.452 & 56,3 & 16.790 & 52,2 \\
\hline & N.D & 57 & 0,6 & 33 & 0,5 & 39 & 1,1 & 16 & 0,3 & 21 & 1,3 & 5 & 0,1 & 2 & 1,0 & 9 & 0,3 & 182 & 0,6 \\
\hline & Total & 8.958 & 100 & 6.086 & 100 & 3.674 & 100 & 4.678 & 100 & 1.648 & 100 & 4.307 & 100 & 210 & 100 & 2.577 & 100 & 32.138 & 100 \\
\hline
\end{tabular}

Fuente: Dane - Estadísticas Vitales, Cuentas Departamentales. Cálculos de los autores 
La conformación de la región Caribe se hizo de acuerdo la división política administrativa de Colombia. Según esta, la región Caribe está conformada por 8 departamentos: Atlántico, Bolívar, Cesar, Córdoba, La Guajira, Magdalena, Sucre y San Andrés y Providencia.

Uno de los temas más importantes a la hora de utilizar el método de capital humano de valoración de la vida humana, es la definición de la tasa de descuento a utilizar. En este estudio se utilizara la tasa promedio encontrada en los trabajos metodológicamente parecidos, que oscila entre el 3\% y 5\%.

Los números que denotan las causas corresponden a la siguiente relación: enfermedades transmisibles (1), neoplastias (2), enfermedades del sistema circulatorio (3), afecciones perinatales (4), causas externas (5), demás causas (6). 